\title{
CFD simulation of micro-particle trapping under water tweezers
}

\author{
Xin-Hua Yi $\cdot$ Xiao-Min Cheng $\cdot$ Feng-Lian Niu \\ Hong-Chao Fan
}

Received: 17 October 2013/Accepted: 20 January 2014/Published online: 2 March 2014

(C) Shanghai University and Springer-Verlag Berlin Heidelberg 2014

\begin{abstract}
In early research, capture and manipulation of particles were mainly achieved by means of light, electricity and plasma in micro-fabrication and micro-assembly. A new method is proposed using micro-water jet to form water tweezers to capture solid particles and implement position control of micro-particles. This paper analyzes the basic principle of water tweezers, and the discrete element method and smoothed particle hydrodynamics method are employed to establish a solid-liquid coupling model used in analyzing the trapping mechanism. A flow field model is set up to simulate dynamic characteristic of water tweezers based on computational fluid dynamics (CFD). Selection of boundary conditions, initial guess, solver control and convergence strategies of the model are discussed. Velocity and pressure of streamline are predicted and discussed under certain input conditions. Simulation results demonstrate that it is an efficiently theoretical method to eventually trap solid particles by water tweezers.
\end{abstract}

Keywords Water tweezers - Micro-particle trapping · Computational fluid dynamics (CFD) - Boundary condition

\section{Introduction}

In the fields of micro-manufacturing and micro-nano manufacturing, assembly will involve incompatible processes,

X.-H. Yi $(\bowtie) \cdot$ X.-M. Cheng · H.-C. Fan

School of Mechanical Engineering, Ningbo University of

Technology, Ningbo 315016, People's Republic of China

e-mail: yixinhua@shu.edu.cn

\section{F.-L. Niu}

School of Mechanical and Electrical Engineering, Ningbo Dahongying University, Ningbo 315175, People's Republic of China complex geometries and different operation mechanisms of manufacturing materials. Its products will depend on the micro-scale positioning, orientation and assembly operations, so that the capture and operation of particles show important aspect in the field of micro-nano manufacturing. Two or three dimensional micro-structural components are realized by the operation of the micro-components relying on the stable capturing of the particles and handling of precise positioning, which becomes the key technology and important base in micro-assembly.

Particle capture and manipulation rely mainly on the magnetic, electric, light, sound $[1,2]$ and suspension technology to implement in the fields of micro-manufacture and micro-assembly. The plasma electromagnetic field method [3] which is used to achieve control of the particles by electromagnetic levitation force has a wide range of researches and applications [4] in the manufacture about the condensation of metal nano-particles. Dielectrophoresis method [5] which denotes the motion of polarizable particles induced by a non-uniform electric field under the influence of electric dipole has been widely used for the operation of the carbon nano-tubes, cells in micro-fluidic protein and DNA separation [6-8] over the past several years. "Optical tweezers" method [9] uses the existence of optically potential wells to make objects float and move relying on visible laser radiation pressure. As a kind of tiny object injury and non-contact manipulation tools, optical tweezers can capture objects suspended in liquid in the diameter from $10 \mathrm{~nm}$ to $10 \mu \mathrm{m}$, and they are widely used in nano-assembly and manipulation of tiny cells and DNA. From the mechanism of particle capture, a conclusion can be drawn that in the excitation of the external energy, the particles can reach a state of equilibrium and be manipulated with changes of excitation force.

By means of particle capture mechanism, water tweezers as a new method of particle trapping has been proposed [10]. 


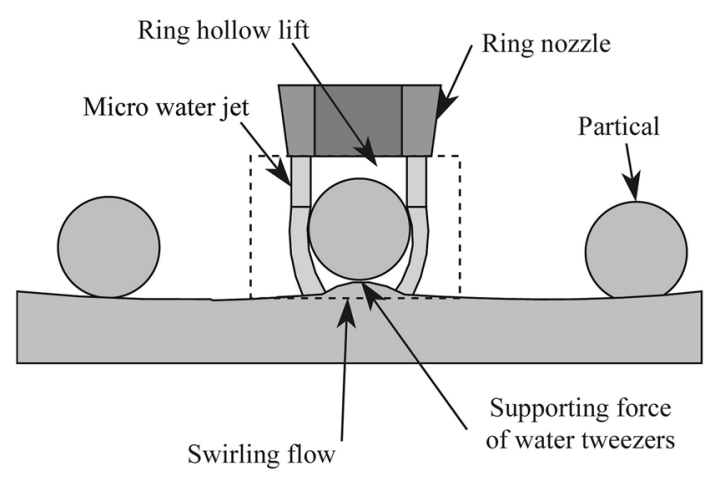

Fig. 1 Principal of water tweezers to capture particles

The aim of water tweezers is to capture stable particles which are between $10 \mu \mathrm{m}$ and $10 \mathrm{~mm}$ in diameter in liquid. The basic principle is that in a certain condition, the ring hollow micro-jet can not wash away the particles, but trap them within the micro-jet which likes tweezers as firmly clamping the particles. The boundary layer is produced between the particles and micro-jet from the leading edge of the particles developing along the particle surface. At the adverse pressure area on the surface of the solid particle where the pressure increases, the boundary layer is separated from the particle surface, and the reverse flow of fluid is forced to generate.

The boundary layer is produced between the particles and micro-jet from the leading edge of the particles, and develops along the particle surface. At the adverse pressure area of pressure increasing surface, boundary layer is separated from the particle surface, and forced to generate reverse flow of fluid. The swirling flow is formed under the lower part of particle. The ring hollow lift and swirling flow will produce the upper supporting on the particles. Supporting force of the swirling flow and the combined effect of the annular lift produces "clamp" effect to the particles, and ultimately form water tweezers. The forming process is shown in Fig. 1.

The experiment research of micro-particle trapping is very complex, hence direct measurement of particle velocities and visualization of particle trajectories are very difficult for the ultrahigh speed and small dimensions. In this paper we mainly discuss the simulation method to analyze the process of micro-particle trapping. This work combines smoothed particle hydrodynamics (SPH) and discrete element method (DEM) to establish the coupling model of solid particle motion under action of water-jet and study the interaction of high-velocity water jet impacting on the particles.

\section{Numerical simulation methods}

\subsection{Theory model of SPH}

In the SPH method, the state of the system uses a series of particles to describe, and these particles contain respective physical parameters, such as density, pressure, velocity and internal energy. According to the conservation equation of particle movement control, the SPH kernel approximation of continuous integral form can be converted to the sum discrete forms of all the particles in support domain. The core of SPH algorithm is the interpolation theory, and it smooths particle through a series of uniform distributions with various physical quantities to solve partial differential equations under various boundary conditions. Grid connection between these particles is not necessary. The continuous form of kernel approximation $f(x)$ can be written in discretized form of a summation of the neighboring particles as follows [11]:

$$
\left\{\begin{array}{l}
f(x)=\sum_{j=1}^{N} \frac{m_{j}}{\rho_{j}} f\left(x_{j}\right) W_{i j}, \\
W_{i j}=W\left(x_{i}-x_{j}, h\right)=W\left(\left|x_{i}-x_{j}\right|, h\right)=W\left(R_{i j}, h\right), \\
R_{i j}=\frac{r_{i j}}{h}=\frac{\left|x_{i}-x_{j}\right|}{h},
\end{array}\right.
$$

where $W_{i j}$ the kernel function which used in the SPH interpolation, $R_{i j}$ the relative distance between $i$ and $j$ particle, $m_{j}, \rho_{j}$ the mass and density of the particle, respectively, $h$ the radius of the approximation domain.

Generally, smooth length is often the same and does not change over time and space in the numerical simulation of fluid density change. But in reality, it results in the increase or decrease of the influence domain of particle due to the deformation which will affect the accuracy of the calculation. For example, the particle number will increase in the effect of the compressive deformation, and the number of particles will decrease in the influence within tensile deformation. There are many ways to acquire smooth length in SPH. In this paper, a very easy way is adopted by using an average density of smooth length transform to update. The most frequently used smoothing function may be the cubic $B$-spine function.

$W\left(s=\frac{|\boldsymbol{r}|}{h}\right)= \begin{cases}C\left(1-\frac{3}{2} u^{2}+\frac{3}{4} u^{3}\right), & |u| \leq 1, \\ \frac{1}{4} C(2-u)^{3}, & 1 \leq|u| \leq 2, \\ 0, & |u| \geq 2,\end{cases}$

where $|\boldsymbol{r}|$ the distance between the two particles, $h$ the smooth length, $C$ the kernel normalization constant, which is equal to $15 \pi h / 7$ for two dimensional simulation and is $3 \pi h / 2$ for three dimensional simulation.

The dimension of the compact support is defined by the smoothing length $h$, and $\left|x_{i}-x_{j}\right|<h$ defines the support domain of the particle. The gradient of the function $f(x)$ is given by differentiating the interpolation function Eq. (2) and can be gotten, 
$\nabla f\left(x_{i}\right)=-\sum_{j=1}^{N} \frac{m_{j}}{\rho_{j}} f\left(x_{j}\right) \nabla W_{i j}$,

where $\nabla W_{i j}=\frac{x_{i}-x_{j}}{r_{i j}} \frac{\partial W_{i j}}{\partial r_{i j}}=\frac{x_{i j}}{r_{i j}} \frac{\partial W_{i j}}{\partial r_{i j}}$.

It can be seen that the gradient function is related to the particle.

\subsection{Coupling of SPH with FEM}

Because the radius of the kernel function $W$ is a constant, the number of FE nodes coupling with SPH particles is limited, as shown in Fig. 2. The FE nodes are added to neighbor using search algorithm of $\mathrm{SPH}$, which results in a list of contact neighbors. In the contact neighbor search algorithm, all FE nodes and SPH particles are considered. This means that any FE node or SPH particle is close enough.

The calculation algorithm [12] of contact force is adapted between the water particle and the FE node of plastic particle. The approach to calculate the contact force vector is adopted to treat the FE nodes as SPH particles. According to the above analytical equations, water velocities in the water jet can be obtained by theoretical method. The coupled FEM and SPH method is implemented by using contact algorithms. The effect of the water particles on the surface of the solid partcle can be aquired by contact type, where water jet is defined with SPH particles and the particles are defined with finite elements. The contact force vector can then be evaluated as [13]

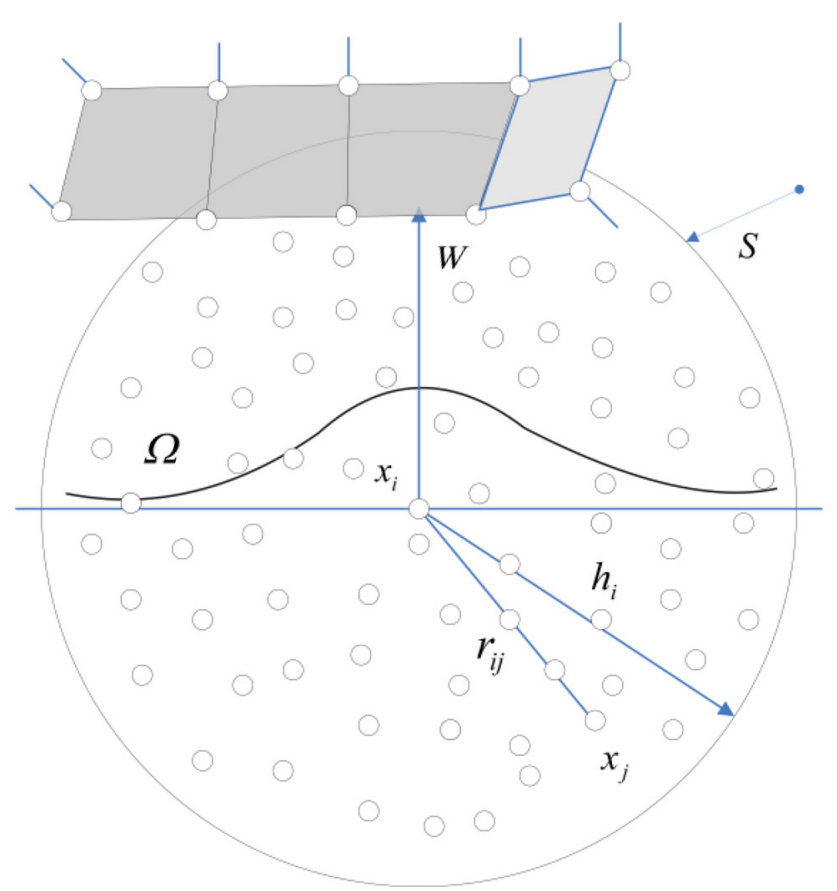

Fig. 2 SPH particle approximations in a two-dimensional problem domain with a surface
$f_{c}\left(x_{i}\right)=\sum_{j}^{A_{\mathrm{NCNR}}} \frac{m_{j}}{\rho_{j}} \frac{m_{i}}{\rho_{i}} K_{n} \frac{W\left(x_{i}-x_{j}\right)^{n-1}}{W\left(h_{\mathrm{Avg}}\right)^{n}} \nabla_{x_{i}} W\left(x_{i}-x_{j}\right)$,

where $A_{\mathrm{NCNR}}$ is the number of nodes or particles that are considered as contact neighbors of node or particle $x_{i}$.

\subsection{Governing equations}

The fluid domain model available is chosen to simulate the present flows. Initially, the CFD model considers three-phase (air, water and plastic particle) flow, where water is treated as the primary phase. The Lagrangian formula of the governing equations is used in the SPH model. The coordinates of particle is $x_{i}$ at the time $t$, and it becomes $x_{j}$ when particle moves to another location. It is the initial function of the coordinates, and object movement Lagrange is described as $x_{j}=x_{j}\left(x_{i}, t\right)$.

The governing equation in this paper is written for solving fluid dynamics problems, and the following equation is used to describe the movement of fluid and status.

Location for the equation of particle motion,

$\frac{\mathrm{d} x_{i}}{\mathrm{~d} t}=v_{i}$.

Conservation of mass equation,

$\frac{\mathrm{d}}{\mathrm{d} t} \rho\left(x_{i}\right)=\sum_{j=1}^{N} m_{j}\left(v\left(x_{j}\right)-v\left(x_{i}\right)\right) W_{i j}$.

Momentum conservation equation,

$\frac{\mathrm{d}}{\mathrm{d} t} v\left(x_{i}\right)=\sum_{j=1}^{N} m_{j}\left(\left(\frac{\sigma\left(x_{j}\right)}{\rho_{i}^{2}}-\frac{\sigma\left(x_{i}\right)}{\rho_{j}^{2}}\right)+\Pi_{i j}\right) W_{i j}$.

Conservation of energy equation,

$\frac{\mathrm{d}}{\mathrm{d} t} E\left(x_{i}\right)=-\sum_{j=1}^{N} m_{j}\left(v\left(x_{j}\right)-v\left(x_{i}\right)\right)\left(\frac{\sigma\left(x_{j}\right)}{\rho_{i}^{2}}+\frac{\Pi_{i j}}{2}\right) W_{i j}+H_{i}$,

where $\sigma$ stress tensor, $E\left(x_{i}\right)$ artificial intern energy unit mass of particle, $\Pi_{i j}$ artificial viscous force, $H_{i}$ artificial heat flow, $W_{i j}$ kernel function.

\section{Numerical simulation of coupling model for water tweezers}

A pure water jet is considered as a two-dimensional, steady axis symmetric turbulent flow which flows through a thin ring nozzle and then goes into the atmosphere as a free jet before entering the water tank. The computational domain includes two coupling surfaces which are water-air coupling surface and solid-liquid coupling surface. When the water jet passes through the solid particle, it is trapped within the center of 

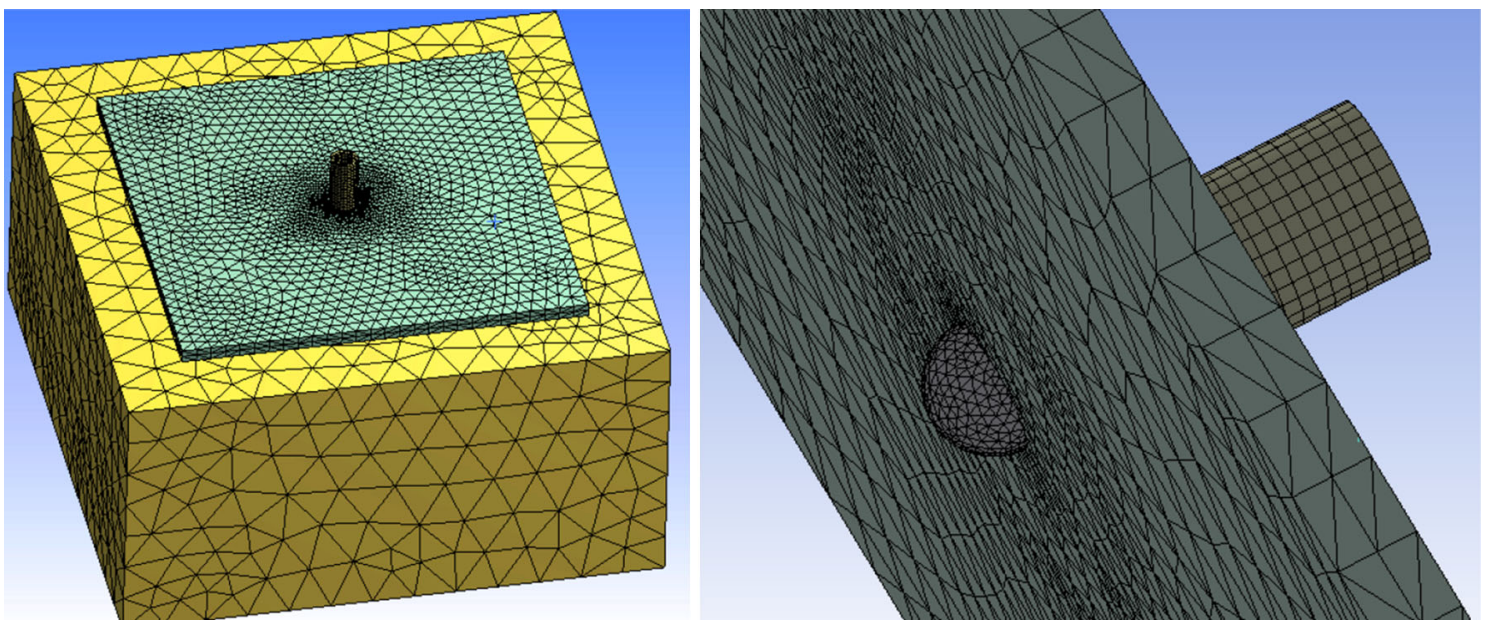

Fig. 3 Discrete finite element model

water jet. The water jet goes another cycle after it goes through the water tanker. The entire flow field model consists of air domain, water basin domain and annular nozzle domain. The dimension of annular nozzle model is inner diameter of $5 \mathrm{~mm}$, outer diameter of $10 \mathrm{~mm}$ and nozzle length of $20 \mathrm{~mm}$. The volume dimension of air domain model is $150 \mathrm{~mm} \times$ $150 \mathrm{~mm} \times 50 \mathrm{~mm}$, and the diameter of particle solid model is $8 \mathrm{~mm}$. The volume dimenstion of flow field model is $200 \mathrm{~mm} \times 200 \mathrm{~mm} \times 100 \mathrm{~mm}$, and the diameter of tanker outlet is $8 \mathrm{~mm}$. In the absence of experimental data, the flow at the nozzle exit is assumed to be fully developed to the $1 / 7$ th power law distribution for the mean velocity profile.

The water jet is modeled by SPH particles, and the target particle is still modeled by discrete elements method (DEM). The jet velocity distribution is supposed as uniformed. The procedure creating the SPH particle model of water jet is explained as follows. In the 3-D FEA model, the water domain, air domain and plastic particle domain are meshed by 6 -node triangular. The micro-water jet domain is meshed by 8-node brick element, as shown in Fig. 3. The water-jet nozzle domain includes 53,553 elements with 12,431 nodes. The plastic particle has 7,200 elements with 8,649 nodes. The mesh of plastic particle will be distorted due to impact of water jet. To simplify the model of water jet, the plastic is set to rigid body.

The default solver values are used for the turbulent kinetic energy and dissipation rate, and the liquid volume fraction is set to one over this boundary. In addition, the water jet in the inlet is assumed to have a negligible velocity defect for the particles. In the CFD model, wall of water domain is treated as a slip wall, whereas air domain is considered as free boundary for which pressure conditions of inlet are used. It is expected that only air will be entrained into the computational domain, therefore the air volume fraction for any fluid entering the domain across section of air domain is set to unity. The geometry of

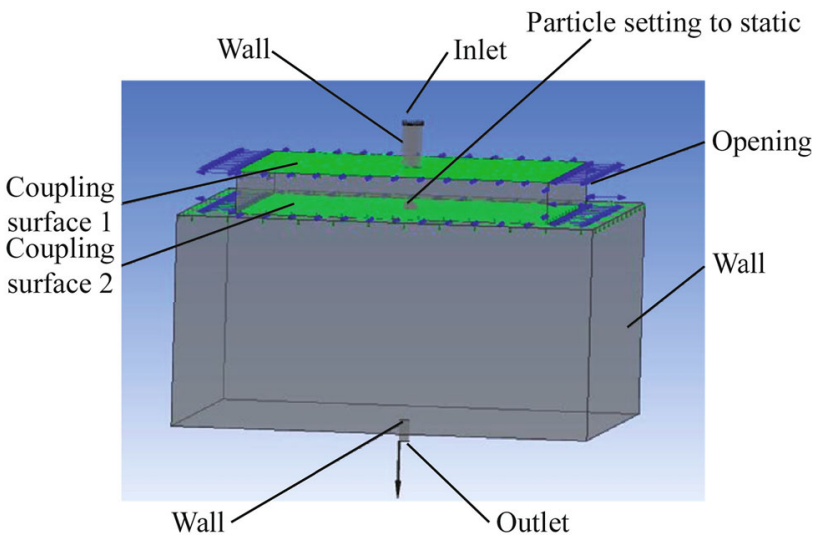

Fig. 4 Computation domain and boundary conditions of CFD model

computational domain with boundary conditions is shown in Fig. 4. The target material is plastics sphere with the diameter of $8 \mathrm{~mm}$, and the water jet with the diameter of $5 \mathrm{~mm}$. Finally, the velocity of the target is $2 \mathrm{~m} / \mathrm{s}$.

The boundaries of the flow field are set as follows:

(i) The nozzle flow, $Q_{\text {Inlet }}=0.3 \mathrm{~kg} / \mathrm{s}$.

(ii) Bottom of the tank outlet flow, $Q_{\text {Outlet }}=0.3 \mathrm{~kg} / \mathrm{s}$.

(iii) Solid particle is set to the static.

(iv) Coupling surface 1 is formed by the nozzle exit and the air domain (seen the top green grid surface ) and coupling surface 2 is formed by the air domain and water domain (see the bottom green grid surface).

(v) The boundary conditions of the air domain are set to "opening".

It is assumed that the solid particles are the static, which ignores the movement of solid particles in the effect of water jet. To form water tweezers under water jet conditions, it is necessary to analyze whether the ring hollow micro jet can achieve capture force. 


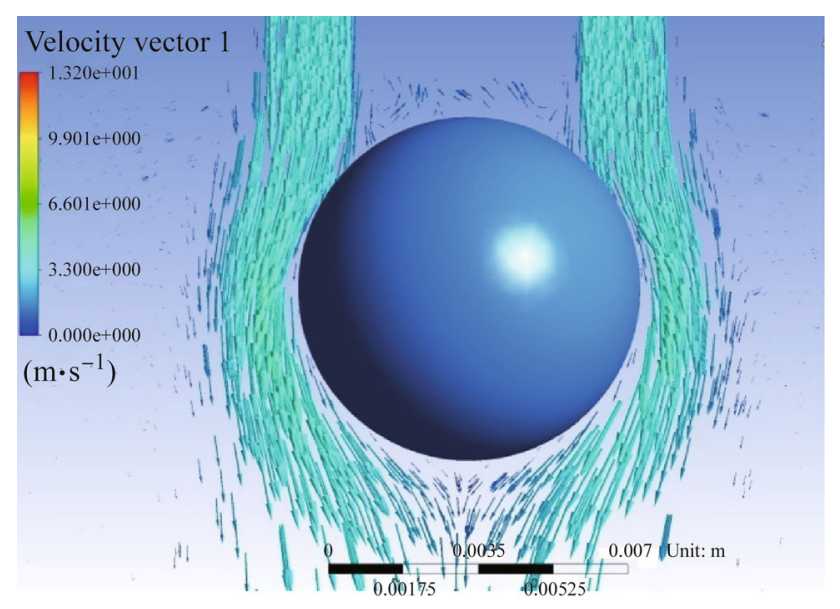

Fig. 5 Direction velocity vector of water jet around the solid particles

\section{Results and discussion}

The direction velocity vector of water flow around the solid particles is shown in Fig. 5. The speed of the particle top is close to zero and two small disturbance vortexes are formed in the effect of water jet. The water jet arrives at the surface of particle and flows along the edge of particle until approaching the bottom of particle to spread. From the pressure gradient around the particle as shown in Fig. 6, a ring hollow lift is formed by pressure gradient whose vector direction is from the bottom to up because of disturbance vortex in the particle upper. In particle bottom, a supported force is created by pressure gradient whose vector direction is from the bottom to up. The force of the water jet acting on the particle is offset in horizontal direction and its function is to balance the particle. The force working in the vertical direction is not zero because of the impact of water jet. To keep the water jet clamping the particle, the supported force or ring hollow lift should be equal or greater than the impact force of water jet.

Figure $7 \mathrm{a}$ shows the velocity change along the streamline when the water particle flows from the inlet to outlet in the working conditions. The velocity of water jet keeps stable in $3.2 \mathrm{~m} / \mathrm{s}$ before the water jet reaches the solid particle. When water jet is approaching the solid particle, the speed seriously decreases because of particles hindering. When the water jet hits on the surface of solids, water jet flows downward along the particle surface to the bottom. Figure $7 \mathrm{~b}$ shows the pressure change along the streamline when the water particle flows from the inlet to outlet in the working conditions. The pressure of water jet keeps in a constant value about $1.02 \times 10^{5} \mathrm{~Pa}$. When water jet is approaching the solid particle, the pressure significantly increases because of particles hindering. After the water jet hits on the surface of solids, water jet flows downward along the particle surface to the outlet and the pressure decreases to a balance pressure about $1 \times 10^{5} \mathrm{~Pa}$ until the water jet goes through the outlet.

As a hypothesis theory about water jet to trap solid particle, it can be seen that the water jet is fully capable of

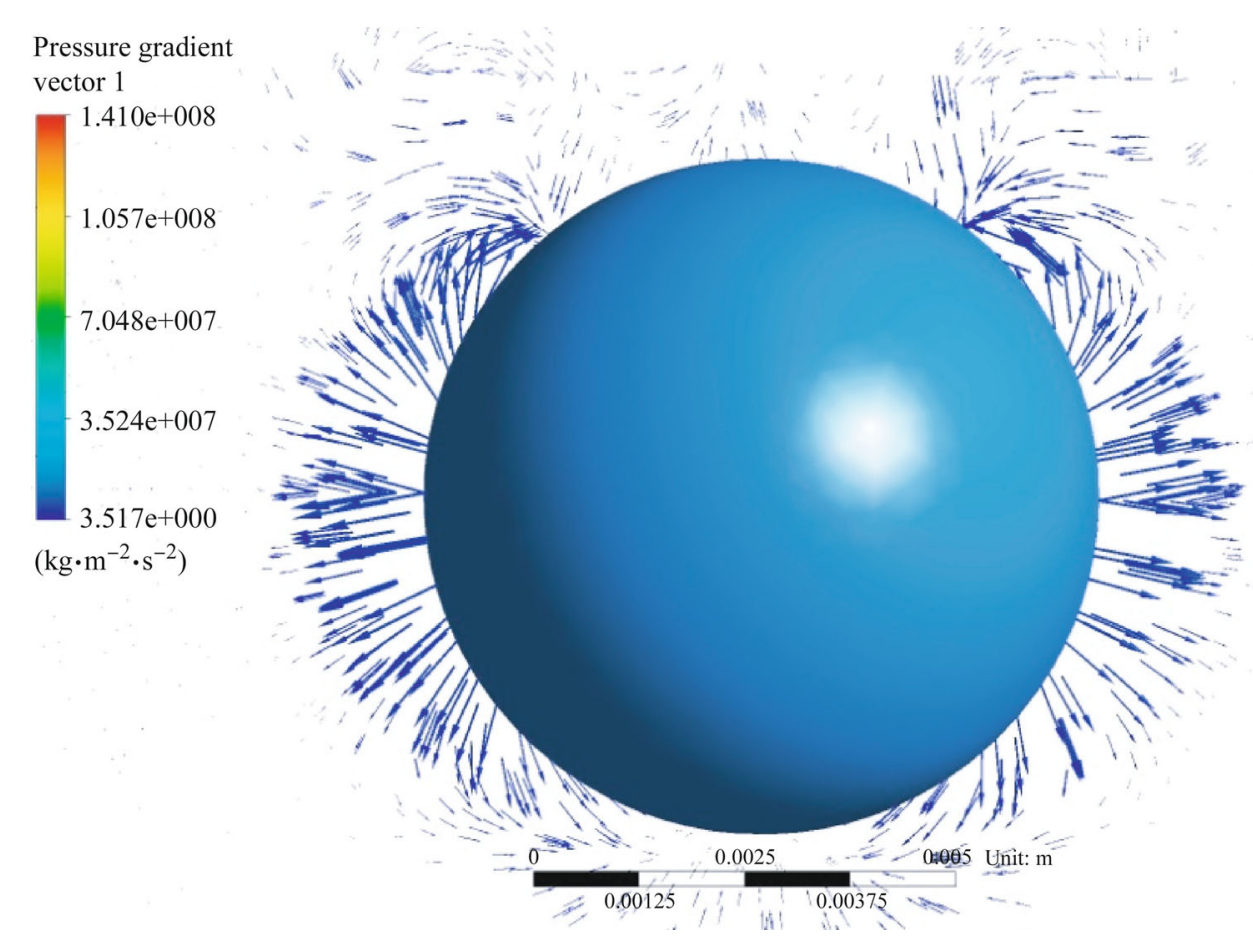

Fig. 6 Direction pressure gradient vector of water jet around the solid particles 

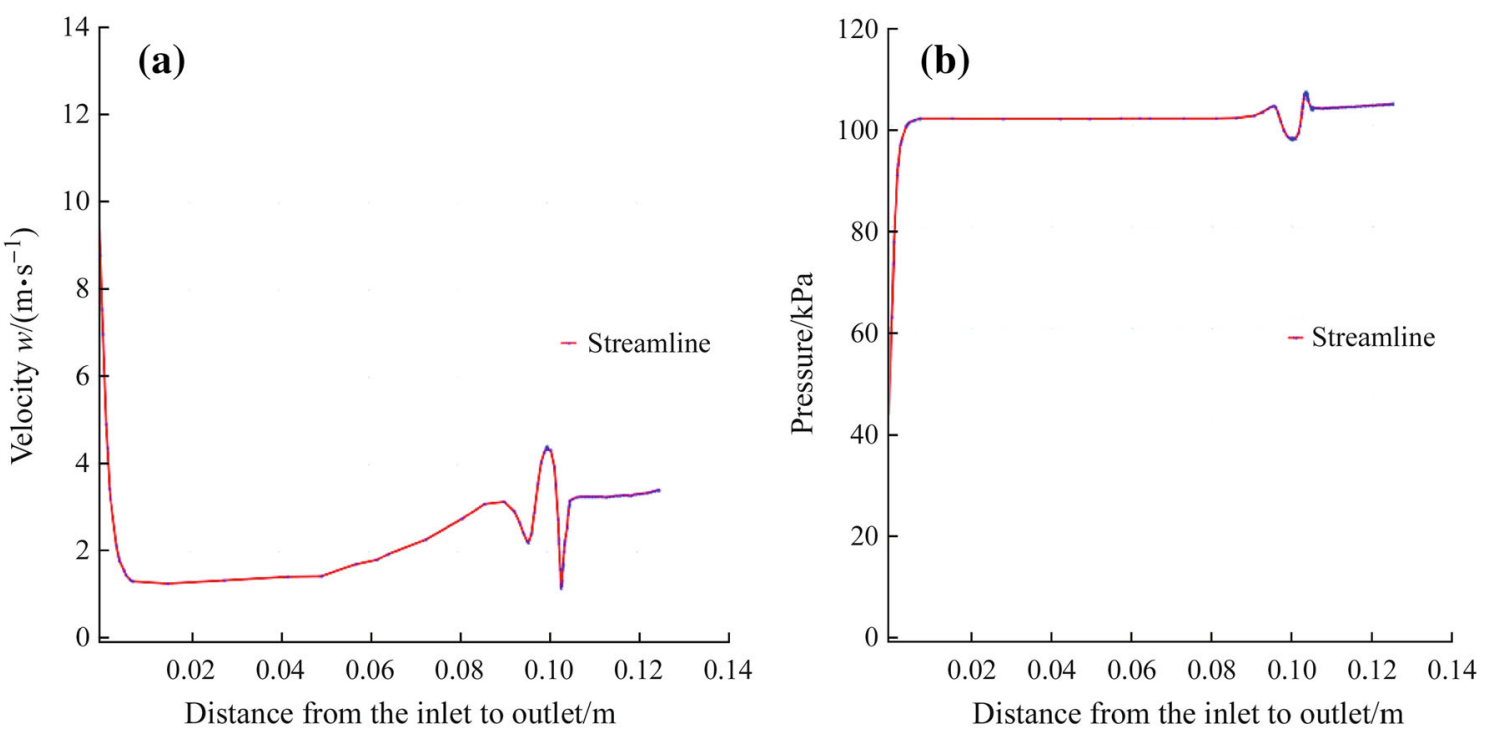

Fig. 7 a Velocity change along the streamline and $\mathbf{b}$ pressure change along streamline

forming the capture of the particle from the simulation results using established CFD simulation model. When the solid particle is in a static state, the formation of water jet flow direction can achieve the clamping capacity on the solid particles from the theoretical analysis results.

\section{Conclusions}

The study has provided an in-depth description of the dynamic characteristics of particles in the action of water jet. A CFD simulation of water tweezers based on water jets has shown a mechanism about how to form water tweezers using micro water jet. A flow field model is set up to analyze the forming process of water tweezers by means of the hybrid coupling of SPH and FEA. The simulation results for the trapping of micro solid particles have demonstrated that micro water jet flow passing ring nozzle can clamp the particle in a certain condition. The velocity and pressure around the particle can provide good condition for the particle trapping. This simulation results are coinciding with the trends extracted from the predictive models. The next study will provide the essential knowledge to optimize the jet characteristics by changing the nozzle design parameters, and mathematical model of the jet and particle characteristics for improving the water tweezers performance to eventually achieve the precise position control of the solid particles using water tweezers.

Acknowledgments This research is jointly sponsored by the National Natural Science Foundation of China (Grant No. 51275251) and the Natural Science Foundation of Ningbo Municipality (Grant No. 2013A610048).

\section{References}

1. Karl F, Ronald S, Fearing KY (2007) Micro-assembly, handbook of industrial robotics. Wiley, Berkeley

2. Fukuda T, Dong L (2003) Assembly of nanodevices with carbon nanotubes through nanorobotic manipulations. Proc IEEE 91: 1803-1818

3. Huang L, Maerkl S, Martin O (2009) Integration of plasmonic trapping in a microfluidic environment. J Opt Express 17:6018-6024

4. Malekzadeh M, Halali M (2011) Production of silver nanoparticles by electromagnetic levitation gas condensation. Chem Eng J 168:441-445

5. Brown K, Westervelt R (2009) Proposed triaxial atomic force microscope contact-free tweezers for nanoassembly. Nanotechnology

6. Shaikh FA, Ugaz VM (2006) Collection, focusing, and metering of DNA in microchannels using addressable electrode arrays for portable low-power bioanalysis. Proc Natl Acad Sci USA 103:4825-4830

7. Chen Z, Wu Z, Tong L, Pan H, Liu Z (2006) Simultaneous dielectrophoretic separation and assembly of single-walled carbon nanotubes on multigap nanoelectrodes and their thermal sensing properties. Anal Chem 78:8069-8075

8. Washizu M, Kurosawa O (1990) Electrostatic manipulation of DNA in microfabricated structures. IEEE $T$ Ind Appl 1990(26): 1165-1172

9. Ashkin A, Dziedzic J, Bjorkholm J, Chu S (1986) Observation of a single-beam gradient force optical trap for dielectric particles. Opt Lett 11:288-290

10. Cheng X, Xu Y, Zhou L (2010) Water tweezers for particles gagging. Int Conf Comput Appl Syst Model 8:22-24

11. Feng Y, Jianming W, Feihong L (2012) Numerical simulation of single particle acceleration process by SPH coupled FEM for abrasive waterjet cutting. Int J Adv Manuf Technol 59:193-200

12. Liu MB, Liu GR (2010) Smoothed particle hydrodynamics (SPH): an overview and recent developments. Arch Comput Methods Eng 17:25-76

13. De Vuyst T, Vignjevic R, Campbell JC (2005) Coupling between meshless and finite element methods. Int $\mathrm{J}$ Impact Eng 31:1054-1064 